\title{
Post vestigia gregum
}

\section{La poétique de l'image dans le commentaire Super Cantica Canticorum de Jean Gerson (1429)}

Dernier ouvrage de Jean Gerson laissé inachevé à sa mort en 1429, le commentaire Super Cantica canticorum se présente comme un double testament, théologique et poétique. L'épithalame y est traité comme un dialogue entre l'âme et Dieu qui fait valoir un «amour de fruition, extatique, anagogique et séraphique, consistant en une perception expérimentale de l'union de l'âme avec Dieu, son objet suprême et sa fin ${ }^{1}$. On a pu y voir un "traité de l'amour de Dieu» ${ }^{2}$ structuré en dix chants ou sympsalmata, à leur tour divisés en «considérations» ou «propriétés», qui couronne la grande entreprise de théorisation gersonienne en matière de théologie mystique ${ }^{3}$.

Je présenterai dans un premier temps la méthode et le modus tractandi suivis par Gerson dans son commentaire, afin de préciser sa place dans la tradition exégétique. Je poserai en particulier la question du lien entre les styles «rhétorique» et «scolastique» distingués dans le prohemium, et du rapport complexe à l'image métaphorique qui en découle. Dans un second temps, j'illustrerai la démarche gersonienne en présentant un extrait de la deuxième partie de l'œuvre, dont je proposerai une analyse littéraire. Le passage, correspondant au deuxième sympsalma et glosant Cant. 1.6-10, forme une unité tant du point de vue de l'idée que de celui de son traitement poétique, marquée par le procédé de l'inclusio (le texte s'ouvre et se referme par le même syntagme, emprunté au verset 6a). J'étudierai comment le concept émerge de l'image (en l'occurrence, une scène pastorale) en s'appuyant sur une vision du sensible dont le traitement se situe aux antipodes de l'ornemental, puis j'examinerai le collage métaphorique avec le motif épique du char de Pharaon, en prêtant une particulière attention à la manière dont le montage creuse l'image et approfondit dans le même temps l'idée théologique sous-jacente.

C'est donc une écriture personnelle, attentive à l'expressivité du poème biblique sans sacrifier aux facilités de l'ornatio rhétorique, une écriture scolastique exploitant le sens figuré du texte mais refusant de se plier à un décryptage allégorique systématique, que je me propose de faire paraître en la rapportant à ses modèles exégétiques et à sa principale source d'inspiration, l'Itinerarium mentis in Deum de Bonaventure.

1 «Amor fruitivus et extaticus vel anagogicus et seraphicus, quia consistit in experimentali perceptione conjunctionis animae cum supremo objecto suo et fine Deo». Je cite le Tractatus super Cantica Canticorum (désormais TCC) d'après Glorieux (1971) 576.

2 Vial (2006) 22.

3 Pour un aperçu du projet gersonien dans son ensemble, tel que le présent commentaire le met en œuvre, voir les pages de Combes (1964) 650-668. 


\section{Novo modo scribere : Gerson dans la tradition exégétique du Cantique des Cantiques}

«Tout est dit et l'on vient trop tard...». C'est sur un constat apparemment désabusé, annonçant la formule de La Bruyère, que s'ouvre le commentaire du Cantique de Gerson. Il n'y a pas là de quoi s'étonner, s’agissant du livre biblique le plus commenté au Moyen Âge, à la «fortune aussi exubérante et encombrante ${ }^{4}$. On comprend les réticences de Gerson à répondre favorablement à la requête qui lui avait été adressée :

\footnotetext{
Verum post tot a summis ingeniis dictata super eisdem Canticis quid aut quale posset addere paupercula nullius vel tepidae devotionis meae, diu satis in dubio fuit animus. Tandem confisus in auxilio precum vestrarum decrevi novum aliquid aut saltem novo modo scribere sub praemisso themate : Amo te. ${ }^{5}$

«Mais quant au type de contribution que pourraient apporter les pauvres ressources de ma faible dévotion - celles de quiconque d'ailleurs - à tant de commentaires composés par les plus grands esprits sur ce Cantique, j'en suis resté longtemps dubitatif. Enfin, m'en remettant à vos prières, je résolus d'écrire quelque chose de nouveau - ou du moins de l'écrire d'une manière nouvelle - sur le thème déjà mentionné : «Je t’aime».
}

La posture est topique. Mais derrière l'humilité affectée du prologue et son pendant obligé, la valorisation implicite des destinataires (les Pères Chartreux qui lui ont commandé le traité), on décèle l'ambition de l'écrivain qui entend, moins par la nouveauté de sa lecture que par ses choix formels et stylistiques, se faire une place dans la tradition exégétique où se sont illustrés avant lui Origène et Grégoire le Grand, Aymond d'Auxerre et Rupert de Deutz, Bernard de Claivaux et Guillaume de Saint-Thierry.

Parmi les critères permettant de situer Gerson dans la longue lignée des commentateurs du Cantique, on retiendra trois éléments. Premièrement, le choix du thema : l'amo te de l'Evangile de Jean (Jn 21.16) où la triple question du Christ à Pierre s'attire trois fois la même réponse («tu sais bien, Seigneur, que je t’aime»). Ce thème ouvre la voie à une réflexion sur l'amour de Dieu dans ses modalités d'énonciation, en tant qu'il essaie de se dire. Or le mot dit tout (amo se lit comme un acrostiche joignant l'alpha et l'oméga - AMO - à la fois principium, medium, finis ${ }^{6}$ ) et pas assez à la fois. Comment extraire alors le sens du signe ? Comment déployer le signifiant en énoncé afin de le mettre en forme d'un point de vue théologique ? Telle est le soubassement de l'enquête gersonienne. L'originalité de Gerson tient en second lieu à sa méthode d'exposition : l'œuvre est divisée en dix chants ou sym-

4 Ricoeur (1998) 411.

5 TCC, 565.

6 «In hac dictione Amo significatur principium, medium et finis secundum tres ejus litteras» (TCC, 593). 
psalmata auxquels correspondent d'une part la structure littéraire du Cantique en dix dialogues ou dragmata, de l'autre les dix cordes du psaltérion de David. Cette divisio textus est probablement empruntée à la Cantuariensis Glossa super Cantica, à moins qu'elle ne soit directement issue de la Glossa tripartita, important commentaire franciscain composé au tournant des XIII ${ }^{e}$ et XIV ${ }^{\mathrm{e}}$ siècles ${ }^{7}$. À chacun de ces dix ensembles correspondent dix «considérations» ou "propriétés» résumées chacune par un distique mnémotechnique. On obtient un total de cinquante unités, assimilables au nombre de jour séparant Pâques de la Pentecôte et aux langues de feu du Saint-Esprit. Telle est la lingua amoris, unifiée et plurielle. Troisième et dernier critère, celui de l'interprétation : Gerson choisit d'orienter son commentaire vers le sens anagogique de l'épithalame ; il y sera donc question du chant d'amour entre l'âme et Dieu, interprété selon les principes de la théologie mystique dionysienne, plus précisément du Pseudo-Denys tel que le transmettent Thomas Gallus et Bonaventure, deux influences qui se combinent aussi dans la Glossa tripartita.

Mais c'est dans la mise en œuvre formelle de son projet de lecture, et dans la poétique qui le sous-tend, qu'il faut chercher la singularité remarquable de Gerson :

Placuit igitur, duce Spiritu Sancto, verarum ope precum coarctare sermonem et aptare principaliter ad sensum anagogicum de amore sponsae. Quod ut sincerius fiat et absque involucris similitudinum, expediens esse censui conditiones vel effectus et signa vel proprietates hujus amoris sub quinquaginta considerationibus, quasi totidem scintillis aut linguis igneis annotare. Verumtamen ne videamur a littera nimis exorbitare, studuimus istas amoris proprietates ab involucris et fumis litterae quadam exagitativa depuratione in emicationem deducere. Hic enim modus tractandi proximior est scholastico quam rhetorico stylo, quali hactenus utique studuimus, permixtione quadam facta ; quam accomode vel utiliter judicet lector, sed benignus. ${ }^{8}$

«C'est pourquoi, guidé par l'Esprit-Saint et avec l'aide d'authentiques prières, il m’a paru bon de resserrer mon discours et de l'adapter principalement au sens anagogique concernant l'amour de l'épouse. Et afin de mener à bien mon projet de manière plus franche, en écartant les voiles de l'analogie, il m'a paru utile de relever les conditions ou les effets, les signes ou les propriétés de cet amour, et de les ordonner en cinquante considérations qui sont comme autant d'étincelles ou de langues de feu. Mais de peur de paraître m'écarter trop loin de la lettre, je me suis attaché à extraire ces propriétés de l'amour de l'enveloppe fumeuse de la lettre, en la soumettant à une intense purification, afin de les faire briller. En effet, cette manière de procéder est plus proche du style scolastique que du style rhétorique tel que je l'ai privilégié jusqu'ici, non sans pratiquer un certain mélange. Au lecteur de juger de la pertinence et de l'utilité de la chose, mais qu'il le fasse avec bienveillance».

Deux éléments retiennent ici l'attention, à commencer par les verbes coarctare et aptare, qui signalent un effort de disposition et de synthèse typique de l'exégèse scolaire, illustré sur le plan formel par les distiques élégiaques accompagnant chaque sympsalma et, sur le plan de l'idée, par la lecture anagogique qui est privilégiée. À cela s'ajoute le motif du voile (involucrum) et de la fumée, qui invite à

7 Cf. Schepers (2000) 85-139.

8 TCC, 577. 
considérer le mode d'agencement du discours (un ensemble de "propriétés» ordonnées en «considérations») comme un instrument de purification et de "clarification» (in emicationem deducere) du langage poétique du Cantique ${ }^{9}$. Il ne s'agit pas de reléguer la lettre du poème au second plan, mais de la rationaliser en mettant en évidence la manière dont le sens émerge de l'image. Tel est le style «scolastique» mis en œuvre ici par Gerson, dans une rupture revendiquée avec le modus tractandi pratiqué auparavant. Rupture plus «rhétorique» - tel est le paradoxe apparent - que réelle (permixtione quadam facta) ? On peut y voir une habile manière d'attirer l'attention du lecteur sur le caractère innovant de la démarche, tout en sollicitant son indulgence.

Gerson ne s'en tient pas là. La question du statut et du traitement des «similitudes» est abordée plus loin, en lien avec la catégorie de lecteurs à laquelle est destiné le commentaire :

Quaererent enim confestim nonnulli nec ab exspectatione sua confundi putabunt : edissere nobis, si expositor es plenarius, quid osculum, quid os, quis osculans, quis osculatus, qualis osculatio ? Dic quae sunt oris duo labia, in osculante simul et osculato. Sed nec terminus hic erit inquisitionis ; fiet processus ad omnes symbolicas significationes quas textus Canticorum decurrit universus : de uberibus, de unguentis, de cellariis, de tabernaculis Cedar, de pellibus Salomonis et ceteris in hunc modum. Sed jam praetacta est nostri super his causa silentii : plus itaque suscepimus umbras et fumos excutere quam discutere. Sane distinctiones et magistrales sermones, homeliae praeterea sanctorum copiosissime pro incipientibus et proficientibus haec docuerunt. Sed nunc perfectis, quamvis imperfectus, debitor sum qui pro consuetudine exercitatos habent sensus. ${ }^{10}$

«Certains n'hésiteraient pas à demander, en croyant ne pouvoir être déçus dans leur attente : «Expose-nous, si tu es un commentateur plus exhaustif que les autres, ce que représentent le baiser, la bouche, l'être qui embrasse et celui qui est embrassée ${ }^{11}$, et ce que signifie l'acte même d'embrasser. Dis-nous ce que sont les deux lèvres de cette bouche, chez celui qui donne le baiser et chez celui qui le reçoit.» Mais il n'y aurait pas de fin à cette enquête et il faudrait en passer par toutes les significations symboliques que compte l'ensemble du texte du Cantique, en commentant de cette manière les seins, les parfums, les celliers, les tentes de Qédar, les peaux des tentes de Salomon etc. Nous avons déjà exposé les raisons de notre silence sur ce point ; c'est pourquoi nous avons pris le parti de dissiper plutôt que de disséquer les ombres et les fumées. Nul doute que les distinctions et les sermons des maîtres, ainsi que les homélies des saints n'aient enseigné avec un luxe de précisions ces interprétations qu'ils destinaient aux débutants et aux plus avancés. Mais pour l'heure, c'est aux parfaits que, tout en étant moi-même imparfait, je suis redevable, eux qui ont leurs facultés aiguisées par la pratique».

9 À noter que la métaphore de l'involucrum est surtout utilisée, depuis la Cosmographie de Bernard Silvestre, pour justifier la fréquentation des lettres païennes, dans une démarche où la poésie, assimilée à une forme d'expression contestable, est valorisée seulement en tant que promesse de vérité. Cf. Iribarren (2011).

10 TCC, 581.

11 On note l'emploi du masculin appliqué aux deux sexes (quis osculans, quis osculatus, et plus loin in osculanto simul et osculato) comme si le genre grammatical participait de l'unification et de la spiritualisation de l'un et de l'autre. Je remercie Arnaud Aizier pour cette remarque et pour sa relecture de ma traduction. 
Les lecteurs familiers du décryptage des «similitudes» systématiquement pratiqué dans les compilations allégoriques du Cantique (on songe au recueil de distinctiones du cistercien Thomas de Perseigne, très diffusé au Moyen Âge ${ }^{12}$ ) s'attendraient à ce que Gerson reprenne à son compte cette technique. Mais il refuse de se livrer à ce qu'il assimile à un vain tour de force (nec terminus hic erit inquisitionis). Son partipris est autre : il se situe moins dans la continuité avec les devanciers qu'à côté, dans une poétique qui révèle ou restaure la vérité de l'image en lui ôtant sans l'altérer le superflu, autrement dit en dissipant (excutere) ce qui la ternit et la dissimule, par opposition à l'image «disséquée» (discutere) et brisée dans la glose allégorique qui analyse le texte terme à terme et se contente de substituer un sens à un autre. La singularité du propos tient à ses destinataires. Loin de se situer dans une démarche pastorale, visant à instruire et à édifier des lecteurs novices ou encore médiocrement aguerris, Gerson s'engage dans un échange avec des "parfaits», autrement dit des contemplatifs déjà formés à ce type de lecture. C'est donc moins un commentaire qu'un «exercice» du texte (exercitium intrinsecum ${ }^{13}$ ) qui leur est ici proposé.

\section{De l'image "évidée» au paysage de l'âme}

Venons-en au passage qui nous intéresse. Situé dans le deuxième sympsalma de la deuxième partie, il correspond aux versets 6 à 10 du chapitre 1 du Cantique :

\footnotetext{
${ }^{6}$ Indica mihi, quem diligit anima mea, ubi pascas, ubi cubes in meridie, ne vagari incipiam post greges sodalium tuorum. ${ }^{7} \mathrm{Si}$ ignoras te, o pulcherrima inter mulieres, egredere et abi post vestigia gregum et pasce hedos tuos juxta tabernacula pastorum. ${ }^{8}$ Equitatui meo in curribus Pharaonis adsimilavi te, amica mea. ${ }^{9}$ Pulchrae sunt genae tuae sicut turturis, collum tuum sicut monilia. ${ }^{10}$ Murenulas aureas faciemus tibi vermiculatas argento. ${ }^{14}$

« ${ }^{6}$ Montre-moi, toi qu'aime mon âme, où tu fais paître, où tu fais reposer à midi, de peur que je ne m'égare en suivant les troupeaux de tes compagnons. ${ }^{7} \mathrm{Si}$ tu ne te connais pas, toi, la plus belle des femmes, sors, suis les traces des troupeaux et fais paître tes boucs près des tentes des bergers. ${ }^{8} \mathrm{~A}$ mes chevaux attelés aux chars de Pharaon je t’ai comparée, mon amie. ${ }^{9}$ Tes joues sont belles comme des tourterelles, ton cou comme des chaînes précieuses. ${ }^{10}$ Nous te ferons des colliers d'or, incrustés de torsades d'argent».
}

À ces six versets correspondent quatre des cinq «propriétés» du deuxième distique élégiaque :

(1) Oppugnatur amor || (2) desiderat alta tenere

(3) Esse vagus refugit || (4) doctus (5) equesque Dei est. ${ }^{15}$

12 PL 206, 17-862. Cf. Bell (2013).

13 TCC, 576

14 On donne le texte dans la version de la Vulgate Sixto-Clémentine.

15 TCC, 577. 
(1) L'amour est combattu ; (2) il cherche à gagner les hauteurs ;

(3) Il répugne à l'errance ; (4) il est savant ; (5) c'est le cheval de Dieu.

On laissera ici de côté la première propriété, qui découle de la précédente et constitue un élément de transition entre le premier et le deuxième sympsalma, et on s'attachera dans un premier temps aux propriétés 2 et 3, qui forment une unité exégético-poétique et dont on présentera successivement les deux composantes, l'une centrée sur l'image du berger-poète, l'autre sur celle du soleil au zénith :

\begin{abstract}
Secunda proprietas. Amor, igitur, desiderat alta tenere. - Indica mihi, inquit sponsa, quem diligit anima mea, ubi pascas, ubi cubes in meridie [Cant. 1.6]. Attendat pietas quemadmodum cito nomen regis, quod est excellentiae et dignitatis, deflexit sensim in nomen pastoris : ubi pascas, inquit. Scimus attribui pastoribus amatoria carmina ubi simplicitas libera et campestris sine dolo versatur, ubi nec arrogantia dominatur. Et quoniam ex hac proprietate pendent non modicum praecedentes et sequentes, consideremus quid sibi velit amor, quid desiderat alta tenere, et tam alta ut notitiam de sponso suo Deo meridianam audeat postulare. ${ }^{16}$

«Deuxième propriété. L'amour cherche à gagner les hauteurs. «Montre-moi, dit l'épouse, toi qu'aime mon âme, où tu fais paître, ou tu fais reposer à midi». Que la piété prête attention à la rapidité avec laquelle le nom de roi, qui exprime l'excellence et la dignité, s'efface devant celui de berger : «où tu fais paître», dit-elle. On prête aux bergers, comme on sait, des chants d'amour caractérisés par leur style simple, fluide et plat, dénué d'artifice et où l'arrogance ne règne pas en maître. Dans la mesure où de cette propriété dépendent en grande partie les précédentes et les suivantes, nous examinerons ce que désire l'amour pour lui-même et quelles sont ces hauteurs auxquelles il aspire, si élevées qu'il ose prétendre à la connaissance de midi au sujet de Dieu son époux».
\end{abstract}

L'attention de Gerson se porte d'abord sur le signifiant. L'enjeu est de montrer qu'il s'agit d'un passage seuil, d'un pivot dans l'interprétation (ex hac proprietate pendent non modicum praecedentes et sequentes) qui se construit à partir de deux images ou figures, celle du berger-poète et celle du soleil de midi.

Voyons d'abord l'image du berger-poète. Le verbe pascas signale un changement référentiel : on glisse sans crier gare d'une image à une autre, de la figure du roi à celle du pâtre. La rupture est camouflée par le texte biblique, qui n'emploie pas le mot pastor, trait dont Gerson signale la subtilité (cito, sensim) : loin d'y voir un collage créant un effet de disparate, il l'interprète comme un détournement doublé d'un abaissement (deflectere). Il signale ainsi une orientation nouvelle donnée au discours qui substitue à l'image royale une image pastorale (les bergers au repos qui chantent l'amour, amatoria carmina) dans laquelle on reconnaît le modèle virgilien des Bucoliques. Ce transfert affectant le dispositif énonciatif s'accompagne d'un changement de registre : on passe d'un style élevé à un sermo humilis (simplicitas libera et campestris) ce qui crée une tension entre l'humilité de la figure du berger et le caractère sublime du «lieu» auquel aspire l'épouse (desiderat alta tenere, et tam 
alta ut...audeat postulare). Gerson ne reconstitue pas les détails de la scène, qui reste relativement abstraite, mais en convoquant les bergers-poètes et en insistant sur l'ethos de leur chant (sine dolo, ubi nec arrogantia versatur), il ramène le texte à un horizon esthétique qui «(é)vide» la représentation de ses détails sensibles jugés pourtant signifiants par les devanciers (c'est le cas des sèmes de nourriture et de repos $^{17}$ ) pour n'en garder qu'un «vestige» sonore.

Le deuxième élément qui retient l'attention de Gerson est le syntagme in meridie. Il s'intègre à l'image précédente par ses connotations : le soleil de midi nous renvoie au moment le plus chaud de la journée, propice au loisir et au chant (on suit le fil de la première Bucolique). Jouant sur la polysémie de la particule ubi, Gerson interprète la question initiale de l'épouse comme portant moins sur le lieu que sur le temps. Il en résulte une temporalité mise en espace, dans laquelle l'image est creusée jusqu'à l'échelle du paysage. Pris au sens spatial (vertical) et considéré dans sa dimension géographique, alta tenere peut s'entendre comme référant aux hauts plateaux (les hauteurs du Golan ?) plus frais à cause de l'altitude, par contraste avec le style campestris ou "plat» référant à la plaine. Au sens temporel, le midi est aussi le moment où le soleil est à son plus haut point dans le ciel, par opposition au matin et au soir.

La distinctio didactique qui suit procède de ce déploiement du sens littéral :

Constat ex doctrina pietatis hausta in sapientiae cellariis quadruplicem in genere esse notitiam amoris divini : meridianam de se et apud se, ubi est qui est ens purum super se reflexum ; meridianam secundo notitiam dicimus de Deo, sed extra se in creatura beata ; tertio matutinam in ipso de creaturis ; quarto vespertinam in proprio genere videndo creaturas per speculum et in aenigmate maxime pro statu viae, vel in speculo et fortassis in aenigmate quodam vestigiorum etiam in patria.

«Il est évident, d'après la doctrine de la piété tirée des celliers de la sagesse, qu'il y a quatre sortes de connaissance de l'amour divin : celle de midi concerne l'amour dans son essence et en lui-même, là où réside celui qui est, l'être pur réfléchi au-dessus de lui-même ; la deuxième sorte de connaissance est aussi de midi et s'applique à Dieu appréhendé hors de lui-même, dans la créature bienheureuse ; la troisième correspond au matin et concerne la connaissance des créatures en Dieu ; la quatrième est celle du soir et consiste à voir les créatures dans leur genre propre à travers un miroir et en énigme particulièrement durant le cours de cette vie, et même dans la patrie, dans le miroir voire dans l'énigme de leurs vestiges».

Le sens spirituel concerne ici la noétique et repose sur des catégories que Gerson emprunte à Bonaventure (Itinerarium mentis in Deum, I.3) qui lui-même les tient d'Augustin (De Genesi ad litteram IV.12) :

17 Voir par exemple l'image du pâturage chez Apponius (Commentaire sur le Cantique des Cantiques II.7-13, De Vregille - Neyrand (1997) 238-253), le lexique de l'innutrition chez saint Bernard (Sermons sur le Cantique, XXXI.10 Verdeyen - Fassetta (1998) 447) dans une interprétation christologique et eucharistique, celle du bon pasteur qui donne sa vie (sa chair) pour nourrir ses brebis, et le développement sur le banquet céleste dans le commentaire vercellien «Deiformis anime gemitus» (cf. Barbet/Ruello (2005) 124-129). 
Haec est igitur via trium dierum in solitudine ; haec est triplex illuminatio unius diei, et prima est sicut vespera, secunda sicut mane, tertia sicut meridies ; haec respicit triplicem rerum existentiam, scilicet in materia, in intelligentia et in arte aeterna [...] haec etiam respicit triplicem substantiam in Christo, qui est scala nostra, scilicet corporalem, spiritualem et divinam.

«Voilà bien la marche de trois jours dans le désert et la triple illumination d'une même journée : la première semblable au soir, la seconde au matin, la troisième au midi. Cette illumination correspond à la triple existence des choses : dans la matière, dans l'esprit et dans l'exemplaire divin [...]. Elle correspond aussi dans le Christ, notre échelle, à sa triple substance : corps, âme et divinité. ${ }^{18}$ »

La source augustinienne s'appliquant à la connaissance angélique ${ }^{19}$, qui n'est pas convoquée ici, on prendra l'Itinerarium pour l'hypotexte le plus probable. Chez Bonaventure, en effet, la connaissance de l'amour de Dieu est déclinée en trois étapes ou degrés, correspondant aux trois moments de la journée, dans une double démarche per / in (chaque degré se dédouble) qui part du sensible, de la créature en tant que vestigium entis, pour s'élever vers le divin (ens purum) en passant par l'âme imago Dei. Gerson laisse de côté l'interprétation christologique de Bonaventure et dédouble le deuxième élément (notitia meridiana) qu'il déplace en première position pour des raisons de mise en valeur rhétorique (c'est l'objet de la quête et de la démonstration) et poétique (ne pas rompre le fil qui relie le sens spirituel à la lettre). Il insiste sur l'intensité du désir d'unité (Vult amor sponsae soli intendere quem ex toto corde non diviso, non disperso diligit) exprimé par le caractère hyperbolique de l'image, ou plutôt - le soleil au zénith n'est pas en soi une hyperbole - l'image ne devient telle que par l'idée qui la sous-tend et l'oriente, à savoir l'aspiration à une forme de connaissance transcendante qui s'énonce et s'actualise en termes affectifs, dans le désir d'union avec l'Un exprimé par l'épouse (Gerson combine ici Origène et Grégoire $^{20}$ ).

D’où la troisième propriété qui, commentant la suite du verset, oppose à ce mouvement vertical, exclusivement orienté vers l'époux divin, les atermoiements d'un désir «divagant», incapable de se fixer sur son objet :

18 Saint Bonaventure, Itinéraire de l'esprit vers Dieu (Duméry (1994) 28-29).

19 Augustin associe la connaissance du matin aux «raisons éternelles» vues par les anges dans le Verbe de Dieu, celle du soir à ces mêmes raisons appréhendées dans les créatures. La distinction est reprise et discutée par Thomas d'Aquin dans sa Somme théologique, $\mathrm{I}^{\mathrm{a}} .58 .6-7$.

20 Il reprend la radicalité intellectuelle d'Origène : «Je ne cherche point d'autres moments, quand tu fais paître le soir, le matin ou au coucher du soleil. Je cherche ce temps-là, quand, dans la splendeur du jour (florente die), tu te trouves en pleine lumière dans l'éclat de ta majesté.» (Homélies sur le Cantique des Cantiques, Rousseau (2007) 97) Il rejoint aussi la dimension affective mise en valeur par Grégoire le Grand : «À midi, le soleil est le plus ardent (sol ferventior est). Quiconque vit d'une foi ardente, vit de l'amour ardent du désir.» (Commentaire sur le Cantique des Cantiques, 41, Bélanger (1984) 131) 
Quidquid enim divisionem ab unitate separantem recipit aut nutrit, horret et amor tamquam unice dilecto contrarium. Propterea sodales hoc loco et greges, similiter et tabernacula pastorum intelligimus in partem uni ovili, uni gregi, uni pastori contrariam accipi.

«Tout ce qui participe de la division séparant de l'unité et qui l'entretient, l'amour l'a en horreur comme étant contraire au seul bien aimé. C'est pourquoi on interprète ici les compagnons et les troupeaux, et semblablement les tentes des bergers, comme désignant des choses contraires à l'unicité de l'étable, du troupeau et du berger».

Loin d'expliquer l'image en en commentant les détails un à un (greges, sodales, tabernacula), Gerson la réduit à une seule idée, l'antithèse unition / dispersion. C'est qu'il lit le Cantique non comme un réservoir de métaphores signifiantes que le poète enfilerait comme des perles et qu'il appartiendrait au commentateur d'élucider tour à tour, mais comme un discours poétique dont le caractère expressif repose sur une combinaison d'images, ainsi que sur des figures alliant connotation et intensification qui lui confèrent sa profondeur sémantique.

\section{Le collage métaphorique}

Les propriétés suivantes $\left(\mathrm{n}^{\circ} 4-5\right)$ rassemblent en deux paragraphes d'inégale longueur les versets 1, 7-10 du Cantique. Elles correspondent d'abord à un changement de locuteur : c'est désormais l'époux qui a la parole. Ce changement n'intervient pas au hasard et participe d'une intention pédagogique :

Doctus est amor sponsae ut altera denotat amoris proprietas et hoc sciens sponsus. Sed ne superbiat sponsa, immo cognoscat qualiter et unde acceperit donum istud, dicit in altera proprietate : si ignoras te, etc. [Cant. 1, 7]..$^{21}$

«L'amour de l'épouse est savant, quand il fait connaître une autre propriété de l'amour, ce qui n'échappe pas à l'époux. Mais de peur que l'épouse n'en conçoive de l'orgueil, et afin qu'elle sache comment et d'où lui vient ce don, il déclare dans une autre propriété : «Si tu ne te connais pas, etc.».

L'enchaînement des répliques, dans le «drame» minutieusement orchestré qu'est le Cantique, relève d'une stratégie de dévoilement du sens qui tient compte des «caractères» et de leur interaction. Là encore, Gerson considère le poème biblique non seulement dans son axe paradigmatique, selon le principe des correspondances analogiques, mais aussi dans son axe syntagmatique, en vertu de quoi il commente le discours tant dans son déroulement syntaxique que dans sa dynamique énonciative ou «dramatique».

De ce point de vue, c'est la prévenance de l'époux à l'égard de l'épouse qui lui fait rajouter ce qui suit ( $5^{\mathrm{e}}$ propriété) :

21 TCC, p. 591. 


\begin{abstract}
Amor est eques Dei, qui vehit scilicet et vehitur. Equitatui meo, etc. [Cant. 1, 8]. Blandiens ergo sponsus neque cum austeritate indignabundus abjiciens : si ignoras te, ait, o pulcherrima mulierum, «si» pro «non» intelligens, egredere et abi, hoc est egredieris et abibis vagabunda post vestigia gregum in dispersione hac illacque discorditer errantium, et illic pasces haedos sensuum tuorum qui ponendi sunt a sinistris sub varietate pastorum fictorum et tabernacula [Cant. 1,7] sibi multiplicantium in falsitate et vanitate. Quod nec feceris aut factura sis, equitatui meo in curribus Pharaoni assimilavi te, amica mea, quia gratiam contemplationis quae te de curribus Pharaonis eripiat, quae persecutrices curas perdere te volentes perdat, largitus sum. ${ }^{22}$

«L'amour est le cheval de Dieu, qui transporte et qui est transporté. «À mes chevaux, etc.» dit l'époux d'une voix douce ; il ajoute posément, d'un ton dénué de sévéritée ${ }^{3}:$ «Si tu ne te connais pas, toi, la plus belle des femmes» (l'hypothèse équivaut à une assertion négative), «sors et suis», c'est-à-dire tu sortiras et suivras en vagabondant «les traces des troupeaux» qui se dispersent et errent en tous sens dans la confusion, et là «tu feras paître les boucs» de tes sens qu'il faut placer à gauche et qui sont sous l'autorité des bergers hypocrites multipliant leurs tentes dans le mensonge et la vanité. Pour t'éviter d'agir ainsi ou de devenir telle, «à mes chevaux attelés aux chars de Pharaon je t'ai comparée, mon amie», car je t'ai accordé la grâce de la contemplation pour t'arracher aux chars de Pharaon et anéantir les soucis qui te harcèlent et veulent ta perte».
\end{abstract}

On assiste en apparence à une nouvelle rupture de registre, avec l'introduction d'une image discordante, celle du cheval attelé au char de combat. Au pastoral succèderait l'épique ; on retournerait au style noble et à la figure royale qui précédaient notre extrait. Or Gerson annule l'écart entre les registres en collant les deux images et en les articulant l'une à l'autre ; il les commente l'une par l'autre, en ce qu'elles s'ajustent comme les deux facettes, opposées mais complémentaires, d'un même raisonnement.

L'enjeu est d'abord dégagé par l'énoncé de la propriété : la relative qui vehit scilicet et vehitur renvoie aux motiones amoris d'Origène, à ces mouvements du désir que le texte met en scène et figure - on rejoint le «nuptial» de Ricœur ${ }^{24}$ - mettant ainsi en valeur la réciprocité ou la réversibilité de la dynamique qui porte la relation. Ces mouvements du désir sont de deux natures opposées. Dans leurs manifestations négatives, ils signifient l'errance et la dispersion ; ce sens est pris en charge par l'image pastorale, dont les connotations sont alors inversées (in dispersione hac illacque discorditer errantium, pastorum fictorum, in falsitate et vanitate) : les bergers sont assimilés au sensible en tant qu'il trompe l'âme et l'égare (vagabunda). Pris comme force positive, les motiones amoris sont assimilés à l'idée d'une course libre et orientée ; ils sont donc du côté de l'image équestre (v. 8) qui se dédouble à son tour en deux facettes antagonistes (le cheval de Salomon vs le char de Pharaon). L'analogie est "prophylactique» (Quod nec feceris aut factura sis, equitatui meo in curribus Pharaoni assimilavi te) en ce qu'elle met en évidence l'intention qui préside

22 Ibid.

23 Réminiscence d'Apponius (blanda commonitione docetur). Op. cit., II.14.255.

24 Op. cit. (note 4). 
au montage des images : il s'agit de prévenir le danger auquel s'expose l'épouse dans l'ardeur de son désir.

L'élément qui articule les deux aspects et introduit le sens spirituel est le syntagme gratiam contemplationis : c'est le «don» de l'époux d'où l'épouse tire sa connaissance (notitia) ; c'est donc aussi le principe moteur de la dynamique «nuptiale». L'exégèse n'est pas neuve. La nouveauté tient ici à son traitement, autrement dit à la manière dont l'idée s'intègre à l'image. La contemplation est associée au domaine céleste et aérien ; elle connote, on l'a vu avec le motif du soleil au zénith, l'élévation ou la hauteur (alta). Saisie du point de vue subjectif, en tant que mouvement qui porte le sujet vers son objet sublime, elle est aussi assimilée à un envol ou à un vol ${ }^{25}$. La connotation est exploitée chez Platon, dans le Banquet 28-29 avec la définition de l'amour comme mouvement d'élévation du sensible vers l'intelligible que donne Diotime, et dans le Phèdre 25 et 34 avec la description de la nature de l'âme, force composée d'un cocher menant un attelage ailé et contrasté : le cheval de droite est de bonne race et obéissant, tandis que celui de gauche est mauvais et rétif. L'image de l'âme-char est connue de la tradition médiévale ${ }^{26}$; elle est employée, en lien avec Cant. 6, 11 (motif du char d'Aminadab) pour figurer l'envol de l'âme vers les réalités supérieures dans le De Isaac d'Ambroise, qui la tient d'Origène ${ }^{27}$. En assimilant l'attelage du Cantique à celui du Phèdre, Gerson figure de manière spatiale et dynamique, par une trajectoire verticale, la polarisation prise en charge par le motif solaire dans la scène pastorale (v. 6-7).

La dynamique tient aussi à la dimension antagoniste de l'image, empruntée elle aussi à Platon : si le bon cheval de l'âme - son désir amoureux - emporte le char vers les hauteurs, le mauvais cheval - celui de gauche, qui représente les passions désordonnées - est responsable de sa chute dans la sensualité de la matière et de son éparpillement dans la «pluralité» des objets qui s'offrent à elle, par opposition à l'unicité de l'époux divin. Rapportée à l'image déjà en place (les bergers et leurs troupeaux sous le soleil de midi), la représentation s'élargit et s'enrichit de l'apport du mythe. Ce faisant, elle subit un autre dédoublement : d'un côté, on visualise l'élévation ascendante de l'âme vers le soleil divin, sous la conduite du cocher (la Grâce) que celui-ci lui a donné ; de l'autre, c'est la chute de l'âme dévoyée par son ou ses chevaux de gauche, assimilés aux boucs de l’Évangile (haedos sensuum tuorum

25 Sur l'importance de cette métaphore chez Gerson, voir Fabre (2013).

26 On la retrouve en particulier chez Martianus Capella (De nuptiis, I) et dans l'œuvre d'Alain de Lille, en particulier dans la description du char construit par les sept Arts et conduit par Raison, qui tient les rênes des chevaux des cinq sens, dans l'Anticlaudianus II.325 à IV.241 (Bossuat 1955) et dans le Sermo de sphaera intelligibili. Cf. D’Alverny (1965) 302.

27 Chez Ambroise (De Isaac vel anima, 8.65, Schenkl - Moreschini, 1982, 106-109), c'est le Christ qui conduit le char, constitué de 2 x 4 chevaux ; les bons chevaux correspondent aux quatre vertus cardinales, les quatre mauvais aux passiones corporis (colère, concupiscence, crainte et injustice). Cf. Courcelle (1975) 599 et Cutino (2016) 13-26. À noter que Gerson consacre quelques lignes au motif du char d'Aminadab au début du Sympsalma IX, mais il ne dit rien des chevaux et se contente de gloser les roues du char, qui représentent les quatre Évangiles (cf. TCC, 628). 
qui ponendi sunt a sinistris $)^{28}$. La dégradation est plaisante et expressive ; elle n'empêche pas de reconnaître le mythe de Phaéton, le mauvais aurige qui, perdant le contrôle du char du Soleil son père, effectue une course erratique au cours de laquelle il manque d'embraser la terre, avant d'être foudroyé par Hélios. Notons que le mythe se retrouve aussi chez Platon, dans le Timée (22d) bien connu au Moyen Âge par le commentaire de Chalcidius.

Le collage intègre ainsi une image dans une autre. Le char grotesque de Pharaon, constitué des bergers et de leurs sinistres troupeaux, a pour pendant l'attelage de la contemplation mené par la Grâce : c'est elle qui maintient le désir unifié de l'âme dans sa «rectitude» et lui permet de tendre vers son but, le soleil divin au zénith. Les éléments mythiques recomposés et redistribués restent certes sous-jacents, mais la vision sensible qui affleure et s'accompagne de l'intériorisation des référents est très expressive. Reste une difficulté, consistant à raccorder à ce qui précède l'évocation des bijoux aux verset 9 et 10 :

\begin{abstract}
Dedi praeterea ut essent pulchrae genae tuae sicut turturis, in honestate conversationis, et collum tuum, quod mihi te conjungit, sit sicut monilia [Cant. 1, 9]. Nos quoque murenulas aureas, quae sunt sententias pietatis aureae, faciemus tibi vermiculatas argento [Cant. 1, 10] eloquentiae, quoniam eloquia mea eloquia casta sunt sicut argentum igne examinatum, probatum terrae, purgatum septuplum. ${ }^{29}$

«'ai aussi fait en sorte que tes joues soient belles comme des tourterelles, par la noblesse de ta vie, et que ton cou qui t'unit à moi soit telle une chaîne précieuse. Quant à nous, ce sont des «colliers d'or», c'est-à-dire des paroles pleines d'une piété resplendissante, que «nous te ferons», des paroles «incrustées des torsades d'argent» de l'éloquence, car mes paroles sont pures, tel l'argent éprouvé au feu, passé au creuset et purifié sept fois».
\end{abstract}

Nouvel effet de disparate ? Plutôt une surimposition d'images, celle de l'attelage et celle du corps de l'épouse. On sait que les chars et les chevaux d'apparat étaient ornés ; quant aux bijoux de l'aimée, ils participent de sa grâce. Gerson les rattache $\mathrm{au}$ «don» qui lui est fait, autrement dit aux effets de la Grâce (l'ultime théorisation de la théologie mystique chez Gerson est une doctrine de la Grâce). Mais le motif ornemental s'entend aussi au plan métapoétique, dans la lettre du commentaire dont l'ambition est d'être l'analogon de son objet, l'amour spirituel. Dire l'amor fruitivus de l'épouse suppose ainsi un discours passé au creuset de l'éloquence (sicut argentum igne examinatum, probatum terrae, purgatum septuplum), un discours "décanté» et intériorisé, ajusté doctrinalement (sententias pietatis aureae) et poétiquement, autrement dit fidèle à la poétique du Cantique telle que Gerson la comprend et la restitue, comme une poétique des "vestiges».

Reste alors, par un effet de mimétique discursive, à clore ce point du commentaire sur lui-même en revenant au point de départ (la petitio de l'épouse en Cant. 
1,6) par la figure typique de la poésie biblique ${ }^{30}$ qu'est l'inclusio, tout en ouvrant le texte sur ce qui suit et, aussi bien, sur ce qui n’a pu être dit :

Sint haec interim pro concordia et sympsalmata secundo contexta absque praejudicio sententiarum quas esse multiplices alias non me latet. Fiat recursus ad petitionem et desiderium sponsae ex amoris fiducia sponso loquentis : amo te. Indica mihi per amorem tuum quo amas me, ubi pascas, ubi cubes in meridie.

«Qu'on prenne dans l'intervalle les considérations suivantes comme visant à rehausser l'harmonie de ce deuxième chant, sans préjuger des explications qui, je ne l'ignore pas, se trouvent ailleurs en grand nombre. Revenons à la demande et au désir de l'épouse qui, mue par la confiance que lui inspire l'amour, dit à l'époux «je t'aime» et «montre-moi» par l'amour dont tu m’aimes «où tu fais paître, où tu fais reposer à midi.»

\section{Conclusion : une poétique des vestigia imaginis}

Si les vestigia gregum du Cantique s'apparentent pour Gerson aux vestigia imaginis de la mystique bonaventurienne, ce sont aussi les «vestiges» de l'image-«similitude» telle qu'elle prolifère dans la lettre et sert ordinairement d'assise au décryptage systématique des compilations allégoriques médiévales. Gerson prend le contrepied de cette démarche en dépouillant l'image de ses détails jugés secondaires et ornementaux, qu'il ne glose pas, pour la «réduire» et n'en garder que la part la plus pure, en la restaurant dans sa vérité. Faut-il y voir un refus ou une mise à distance de la rhétorique ? Gerson met en œuvre, de son propre aveu, un modus tractandi qui s'appuie sur les «règles scolastiques de la théologie et de la philosophie» (scholasticis theologiae et philosophiae regulis ${ }^{31}$ ) et s'écarte du «style rhétorique» qu'il a jusque là privilégié. Ce n'est pas pour autant un rejet de la rhétorique, dont il s'est efforcé de promouvoir le bon usage à l'université, mais un changement de méthode induit par une nouvelle intentio. En entreprenant de ramener le Cantique à une «intelligence commune» qui est celle du «mystère» que les «parfaits» seuls peuvent comprendre, intérioriser et "pratiquer» tel un exercice spirituel (exercitium intrinsecum), Gerson renonce à une visée homilétique et édifiante destinée au plus grand nombre. C'est dans ce dernier cadre que la rhétorique classique par son usage des figures et des ornements (expolitio) vient à l'appui de la démonstration en s'adressant aux affects in declamationibus et exhortationibus moralibus sine fine ${ }^{32}$. En cela aussi Gerson se démarque de l'horizon homilétique qui est le fait de nombreux commentaires, tant monastiques que scolaires ; il s'écarte aussi de la perspective pastorale qui était celle du De duplici logica. Dans cet opus ultimum qu'est le traité

30 Cf. Alter (2003).

31 TCC, p. 579.

32 Ibid., p. 576. 
sur le Cantique, la rhétorique n'est certes pas évacuée, mais en changeant d'objet et de visée, elle a changé de nature.

\section{Bibliographie}

Alter (2003): Robert Alter, L'art de la poésie biblique, trad. fr. par Ch. Leroy et J.-P. Sonnet, Bruxelles, Editions Lessius, 2003.

Barbet/Ruello (2005): Un commentaire vercellien du Cantique des Cantiques «Deiformis anime gemitus», Jeanne Barbet (éd.) et Francis Ruello (trad.), Turnhout, Brepos, 2005.

Bélanger (1984): Grégoire le Grand, Commentaire sur le Cantique des Cantiques, trad. R. Bélanger, Paris, Cerf: «Sources chrétiennes, 314», 1984.

Bell (2013): David N. Bell, « Le Commentaire du Cantique des Cantiques de Thomas de Perseigne revisité ॥, in: Annales de Bretagne et des Pays de l'Ouest [En ligne], t. 120/3 (2013), mis en ligne le 30 septembre 2015, consulté le 08 janvier 2017

Bossuat (1955): Alain de Lille, Anticlaudianus, Robert Bossuat (éd.), Paris, Vrin, 1955.

Combes (1964): André Combes, La théologie mystique de Gerson. Profil de son évolution, Rome etc, Desclée \& Cie, 1964, t. II.

Courcelle (1975): Pierre Courcelle, "Connais-toi toi-même» de Socrate à saint Bernard, Paris, Etudes augustiniennes, 1975, t. III.

Cutino (2016): «Ambroise de Milan et l'exégèse vétérotestamentaire d’Origène: questions méthodologiques à partir d'un cas exemplaire», in: Transmission et réception des Pères grecs dans l'Occident, de l'Antiquité tradive à la Renaissance. Entre philologie, herméneutique et théologie, Emanuela Prinzivalli, François Vinel et Michele Cutino avec la collaboration d'Isabelle Perée (éds.), Paris, Institut d'Etudes Augustiniennes, 2016, p. 13-26

D’Alverny (1965): Alain de Lille, Textes inédits, Marie-Thérèse d’Alverny (éd.), Paris, Vrin, 1965.

De Vregille - Neyrand (1997): Apponius, Commentaire sur le Cantique des Cantiques, t. I, (livres I-III), édition et traduction par Bernard de Vregille et Louis Neyrand, Paris, Cerf: «Sources chrétiennes, 420 », 1997.

Duméry (1994): Saint Bonaventure, Itinéraire de l'esprit vers Dieu, texte de Quaracchi, traduction par Henry Duméry, Paris, Vrin, 1994.

Fabre (2013): Isabelle Fabre, «La plume et l'envol: une analyse stylistique de la Méditation sur l'Ascension de Jean Gerson», in: Revue de l'Histoire des Religions, numéro spécial "Sermo mysticus», Cédric Giraud et François Trémolières (dir.), t. 230, 4/2013, p. 509-544.

Glorieux (1971): Palémon Glorieux (éd.), Jean Gerson, Euvres complètes, Paris, Desclée \& Cie, 1971, t. VIII, $\mathrm{n}^{\circ} 422$ (Super Cantica Canticorum).

Iribarren (2011): Isabel Iribarren, "Le théologien: poète de la doctrine. Quelques considérations autour de la Josephina de Jean Gerson », in: Revue des sciences religieuses [En ligne], 85/3 (2011), mis en ligne le 12 mars 2015, consulté le 01 octobre 2016

Ricoeur (1998): Paul Ricoeur et André LaCocque, Penser la Bible, Paris, Seuil, 1998.

Rousseau (2007): Origène, Homélies sur le Cantique des Cantiques, trad. Olivier Rousseau, Paris, Cerf: «Sources chrétiennes, 37bis», 2007.

Schenkl - Moreschini (1982): Ambroise, De Isaac vel anima, Carolus Schenkl (éd.), Claudio Moreschini (trad.), Milan/Rome, Bibliotheca Ambrosiana/Città Nuova Editrice: «Sancti Ambrosii Episcopi Mediolanensis Opera, 3», 1982.

Schepers (2000): Kees Schepers, «The Genesis of Glossa Tripartita super Cantica», in: Revue d'histoire des textes, 29 (1999), 2000, p. 85-139.

Vial (2006): Marc Vial, Jean Gerson théoricien de la théologie mystique, Paris, Vrin, 2006. 